\title{
MODELO COMPUTACIONAL ESTOCÁSTICO PARA SIMULAÇÃO DE SÉRIES CLIMÁTICAS DIÁRIAS DE UMIDADE RELATIVA DO AR, BASEADO NA PARAMETRIZAÇÃO DINÂMICA DAS DISTRIBUIÇÕES DE PROBABILIDADE DECORRENTE DA RETROALIMENTAÇÃO DE DADOS
}

\author{
Jorim Sousa das Virgens Filho ${ }^{34}$ \\ Maysa de Lima Leite 35 \\ Jaqueline Rissa Franco $^{36}$ \\ Monica Korelo ${ }^{37}$
}

\begin{abstract}
RESUMO
A umidade relativa do ar como um fator climático é indispensável em diversos processos físicos e biológicos, relacionados com as atividades humanas. Sua ocorrência em maior ou menor grau pode, por exemplo, interferir na saúde dos seres humanos, proporcionar efeitos desejáveis ou não no crescimento de plantas, auxiliar na previsão de incêndios florestais, ser decisiva na incidência de pragas e doenças, tanto em animais como em plantas, bem como no conforto térmico das instalações de animais confinados. Sendo assim, este trabalho teve como principal objetivo o desenvolvimento de um modelo computacional estocástico para simulação de séries climáticas diárias de umidade relativa do ar, baseado na parametrização dinâmica das distribuições de probabilidade, decorrente da retroalimentação de dados. Foram utilizadas séries climáticas de umidade relativa do ar, onde se empregou o modelo da distribuição Beta para dias considerados secos e o modelo da distribuição Normal para dias considerados chuvosos. Utilizou-se a linguagem de programação $\mathrm{C}++$ Builder no desenvolvimento do modelo que é uma linguagem de alto nível orientada a objetos e adequada para o gerenciamento de banco de dados. As séries geradas se mostraram muito próximas, realisticamente, das séries observadas, apresentando uma leve tendência de superestimação das mesmas. Espera-se que quando utilizado conjuntamente em outros modelos, o mesmo se apresente como uma alternativa viável para simular a umidade do ar no cenário ambiental.
\end{abstract}

Palavras-chave: umidade relativa do ar, simulação de dados climáticos.

\section{ABSTRACT}

The relative humidity as a climatic factor is essential in many biological and physical processes, related to human activities. Their occurrence in a bigger or

\footnotetext{
${ }^{34}$ Doutor em Agronomia, Professor Adjunto, Depto de Informática - UEPG - Ponta Grossa-PR.

2 Doutor em Agronomia, Professor Adjunto, Depto de Biologia Geral - UEPG - Ponta Grossa-PR.

${ }^{3}$ Acadêmica do curso de Engenharia de Computação - UEPG - Ponta Grossa-PR.

${ }^{37}$ Acadêmica do curso de Engenharia de Computação - UEPG - Ponta Grossa-PR.
} 
smaller degree can interfere with human health, to provide desirable effects or not in the growth of plants, to assist in the prediction of forest fires, to be decisive in the incidence of pests and diseases, both in animals and in plants, as well as in the thermal comfort of the facilities of the confined animal. Thus, this work had as main objective the development of a stochastic computational model for simulation of daily climatic series of relative humidity, based on dynamic parameterization of probability distributions, arising from the feedback data. Climatic series of relative humidity were employed, besides the use of the probabilistic model of Beta distribution for days considered dry and the model of Normal distribution for days considered rainy. The programming language $\mathrm{C}++$ Builder was used to develop the model which is a high-level language oriented to objects and appropriate for managing the database. The generated series were very close, realistically, of the observed series, showing a slight tendency to overestimate them. It is expected that when used together in other models, it presents as an viable alternative to simulate the relative humidity in the environmental scenario.

Key-words: relative humidity, simulation of climatic data.

\section{INTRODUÇÃO}

O vapor d'água não pode ser considerado um componente normal da atmosfera, pois, por definição, a composição atmosférica refere-se a ar seco e limpo. Entretanto é uma constatação que o ar sempre contém certa quantidade de vapor d'água, que do ponto de vista meteorológico ou climatológico, faz com que o ar expresse uma propriedade muito importante que é a sua umidade.

Segundo Tolentino et al (2004), o teor desta umidade do ar é expresso de uma maneira geral como umidade absoluta ou como umidade relativa. Esta última por sua vez, indica a razão (geralmente expressa em porcentagem) entre a quantidade efetiva de vapor no ar e a quantidade máxima de vapor d'água, que a mesma quantidade de ar poderia conter, se estivesse saturada dessa substância, em determinada temperatura.

A umidade relativa do ar como um fator climático é indispensável em diversos processos físicos e biológicos, relacionados com as atividades humanas. Sua ocorrência em maior ou menor grau pode, por exemplo, interferir na saúde dos seres humanos, principalmente nos casos de doenças do aparelho respiratório (SOUZA e SANT'ANNA NETO, 2008), gerando muitos problemas para saúde pública.

Em estudos agroclimatológicos como os abordados em Caliman et al (2005), Beltrão et al (2003) e Cola et al (2009), o conhecimento do seu comportamento, segundo Mota (1981), pode ter no mínimo dois efeitos benéficos possíveis no crescimento das plantas que é a absorção direta da umidade do ar saturado e o aumento do nível fotossintético das plantas na presença da umidade sob a ação da luz. Além disso, o seu monitoramento auxilia no manejo das atividades florestais, possibilitando antever riscos de incêndios, melhorando assim o manejo da atividade diminuindo custos de produção e proteção ambiental, contribuindo para a sustentabilidade da silvicultura.

Em pesquisas bioclimatológicas, como as apresentadas em Neiva et al (2004) e Turco et al (2006), o nível com que a umidade relativa do ar se 
manifesta é decisiva na incidência de pragas e doenças, tanto em animais como em plantas, bem como na qualidade de produtos agropecuários e no conforto térmico das instalações de animais confinados.

A interação de componentes probabilísticos com modelos de outros componentes de um agroecossistema, deve fornecer um método para medir o risco de incerteza climática que está relacionado com gerenciamentos alternativos de empreendimentos agrícolas. Além disso, partindo da premissa que as produções agrícolas podem ser consideradas elementos probabilísticos, no sentido de que dependem dos elementos climáticos durante a época de crescimento de uma cultura, torna-se importante a elaboração de modelos de simulação que gerem dados sintéticos de clima, com a finalidade de reproduzir o comportamento das distribuições de probabilidades desses componentes climáticos.

Segundo Hoogenboom (2000), dados climáticos históricos são importantes para todas as aplicações de modelagem de culturas agrícolas, exceto para aquelas relacionadas com previsões e prognósticos. Porém muitas vezes estes dados não estão disponíveis ou os registros são de períodos curtos ou ainda limitam-se a registros de dados termopluviométricos. Nestes casos, geradores de dados climáticos podem ser usados para gerar sequências diárias para as mais diversas variáveis climáticas, como entrada para modelos agronômicos. Assim, muitos desses modelos como o CROPGRO e o CERES, por exemplo, incorporaram esses geradores de clima como uma das etapas na modelagem para sistemas de apoio à decisão.

Richardson (1985), utilizando dados climáticos diários simulados pelo modelo WGEN (RICHARDSON e WRIGHT, 1984) em substituição à dados climáticos reais como entrada para um modelo de crescimento para a cultura do trigo, não constatou diferenças significativas nos resultados obtidos, mostrando que um gerador de dados climáticos acoplado a um modelo de crescimento para uma cultura, fornece um método bastante eficiente para avaliar os riscos de produção com opções de gerenciamento específico para locais selecionados.

Semenov e Barrow (1997) desenvolveram o LARS-WG, um gerador estocástico de dados climáticos, com o objetivo de avaliar os efeitos das mudanças climáticas sobre o potencial e 0 risco agrícola. Os autores reproduziram alterações no cenário climático para algumas cidades da Europa e, concluíram que alterações na variabilidade dos elementos climáticos poderiam afetar mais profundamente o rendimento das culturas e a probabilidade de eventos climáticos extremos, do que simples alterações nos valores médios.

Bannayan et al. (2003), numa aplicação do modelo CERES-Wheat para simulação da cultura do trigo de inverno no Reino Unido, constataram que os dados climáticos simulados pelo gerador SIMMETEO forneceram uma previsão realística da produtividade da cultura em substituição ao dados observados.

Kuchar (2004) avaliou o WGENK, uma versão modificada do WGEN, na simulação de dados climáticos para uma localidade do sudoeste da Polônia e encontrou pequenas diferenças entre dados simulados e observados.

No Brasil, Virgens Filho (1997) idealizou num primeiro momento o GEPAC Gerador Estocástico de Parâmetros Climáticos, um modelo computacional para simulação de dados diários de precipitação pluviométrica, radiação solar global, temperatura (máxima e mínima) e umidade relativa do ar. Com o intuito de melhorar a qualidade dos dados simulados, o autor desenvolveu o gerador SEDAC_R - Simulador Estocástico de Dados Climáticos que simula dados de precipitação pluviométrica, radiação solar global e temperatura do ar (Máxima e 
Mínima) a partir de uma metodologia baseada num algoritmo de parametrização dinâmica das distribuições de probabilidade, decorrente da retroalimentação de dados (VIRGENS FILHO, 2001). É importante destacar que neste modelo não foi incluída a variável climática umidade relativa do ar.

Tendo em vista a grande importância da umidade relativa do ar como fator climático dentro de uma perspectiva sustentável das atividades humanas, este trabalho teve como principal objetivo o desenvolvimento de um modelo computacional estocástico para simulação de séries climáticas diárias de umidade relativa do ar, baseado na parametrização dinâmica das distribuições de probabilidade, decorrente da retroalimentação de dados.

\section{MATERIAL E MÉTODOS}

Este trabalho foi desenvolvido junto ao Laboratório de Computação Científica do Departamento de Informática da Universidade Estadual de Ponta Grossa. Para tanto, foram utilizados dados diários de umidade relativa do ar para a localidade de Ponta Grossa-PR, cuja estação meteorológica, pertencente ao Instituto Agronômico do Paraná - IAPAR tem as seguintes coordenadas geográficas: Latitude 25013'S; Longitude $50^{\circ} 01^{\prime} \mathrm{W}$; Altitude $880 \mathrm{~m}$. A série climática de umidade relativa do ar compreendeu um período de 48 anos (19542001), dos quais 24 anos (1954-1977) foram utilizados para parametrizar os modelos probabilísticos e 24 anos (1978-2001) para validar os dados simulados.

Os modelos probabilísticos para geração das séries climáticas de umidade relativa do ar foram condicionados à ocorrência de dias chuvosos, ou seja, dias com precipitação pluviométrica. As probabilidades de ocorrência de dias chuvosos foram estimadas mensalmente, para as localidades avaliadas, por meio do processo estocástico cadeias de Markov. A primeira ordem da cadeia de Markov, com apenas dois estados, foi estimada conforme definição em Virgens Filho (2001).

Expressando uma série climatológica de dados diários de umidade relativa do ar para um determinado mês, como um valor decimal de " 0 a 1 " e considerando-a contínua neste intervalo, Yao (1974) concluiu que a freqüência das observações segue uma distribuição Beta. Porém Sediyama et al. (1978), verificou que em dias chuvosos o ajuste não é satisfatório, visto que existe uma alta variância causada pelo registro de valores com baixa umidade relativa do ar, mesmo nos dias com alguma precipitação pluviométrica. Diante disso, nesta pesquisa considerou-se que os dados de umidade relativa do ar para dias secos foram representados pela distribuição Beta cujo modelo probabilístico tem a seguinte função de densidade de probabilidade:

$$
f(x)=\frac{\Gamma(p+q)}{\Gamma(p) \Gamma(q)} x^{p-1}(1-x)^{q-1}
$$

onde o parâmetro de forma "p" é estimado pelo método dos momentos definido em Larsen e Pense (1982) por:

$$
\mathrm{p}=\mathrm{w}-\mathrm{v}(1+\mathrm{w})^{2}
$$

onde: 


$$
\begin{aligned}
& v=\frac{\sum_{i=1}^{i=n}\left(x_{i}-\bar{x}\right)^{2}}{n-1} \\
& \bar{x}=\frac{\sum_{i=1}^{i=n} x_{i}}{n} \\
& w=\frac{1-\bar{x}}{\bar{x}}
\end{aligned}
$$

sendo que " $\mathrm{x}$ " é $\mathrm{o}$ valor da umidade relativa do ar em porcentagem no intervalo $[0 ; 1]$.

O parâmetro de escala " $q$ " é estimado por meio de:

$$
q=p w
$$

Para dias considerados chuvosos (com precipitação pluviométrica maior que $0 \mathrm{~mm}$ ), foi definido que os desvios dos dados reais em relação ao menor valor da umidade relativa do ar dentro de cada mês, foram representados pelo modelo probabilístico da distribuição Normal, conforme avaliação prévia realizada em Virgens Filho (1997). Desta forma, o tratamento dos dados de umidade relativa do ar para dias úmidos, foi realizado da seguinte maneira :

onde :

$$
\text { TUCP }=\text { UR }- \text { MINUR }
$$

TUCP = Valores transformados de umidade relativa do ar para dias chuvosos; $\mathrm{UR}=$ Valores de umidade relativa do ar para dias chuvosos;

MINUR = Menor valor de umidade relativa do ar dentro de cada mês para dias chuvosos.

A distribuição Normal, utilizada nesta pesquisa tem a seguinte função de distribuição:

$$
f(x)=\frac{1}{\sigma \sqrt{2 \pi}} e^{-\frac{(x-\mu)^{2}}{2 \sigma^{2}}}
$$

onde :

$$
\sigma=\sqrt{\frac{\sum_{i=1}^{n}\left(x_{i}-\bar{x}\right)^{2}}{n-1}}
$$




$$
\mu=\frac{\sum_{i=1}^{n} x_{i}}{n}
$$

sendo que: $x=$ TUCP

A metodologia utilizada na simulação dos valores de umidade relativa do ar foi concebida a partir do princípio de que, ao se fixar um período base de dados históricos, o mesmo se move no tempo à medida que a simulação estiver sendo executada. Desta forma, cada ano simulado foi adicionado à série histórica da qual se descartou o ano mais remoto. É importante destacar que a cada movimento do período base no tempo, os parâmetros das distribuições de probabilidade são novamente estimados, fazendo com que, embora a distribuição de probabilidade tenha novos parâmetros, a duração do período permaneça a mesma.

Sendo assim, por exemplo, se o período base for de 1979 a 2008 (30 anos) e se desejar simular o período de 2009 a 2013, tem-se o seguinte comportamento do sistema:

_ Na simulação de 2009 o período base seria 1979-2008;

_ Na simulação de 2010 o período base seria 1980-2009;

- Na simulação de 2011 o período base seria 1981-2010;

_ Na simulação de 2012 o período base seria 1982-2011;

_ Na simulação de 2013 o período base seria 1983-2012.

É interessante observar que pela metodologia convencionalmente utilizada, por mais que se desejasse simular períodos futuros, os anos simulados teriam como base o mesmo período (1979-2008) e consequentemente os mesmos parâmetros estimados da distribuição de probabilidade. Está técnica já foi avaliada e validada com outras variáveis climáticas em Virgens Filho (2001).

O modelo computacional para simulação de dados climáticos de umidade relativa do ar foi desenvolvido utilizando a linguagem de programação Borland $\mathrm{C}++$ Builder, que é uma linguagem de alto nível orientada a objetos e adequada para o gerenciamento de banco de dados. A ferramenta computacional foi projetada e idealizada para ser executada numa plataforma "Windows", gerando arquivos com dados simulados no formato "Texto" que poderão ser acessados posteriormente por outros aplicativos e planilhas eletrônicas.

$\mathrm{Na}$ execução da parametrização dos dados foram requeridos além da data e o respectivo valor observado da umidade relativa, as condições " 1 " para dia chuvoso, "0" para dia seco ou ainda o valor da precipitação pluviométrica medida em $\mathrm{mm}$.

Para a geração da variável aleatória com distribuição Beta, foi utilizado o algoritmo descrito em Bustos e Frery (1992). Na geração da variável aleatória com distribuição Normal, foi implementado o método de Marsaglia-Bray apresentado por Shannon (1975). Para a geração do elemento estocástico nos modelos probabilísticos, foi inserido o algoritmo para geração de números pseudo-aleatórios desenvolvido por Wichmann \& Hill (1982). Este gerador é baseado no método congruencial multiplicativo, que segundo os autores, passou por uma infinidade de testes de aleatoriedade apresentando excelente 
desempenho. O tamanho do seu ciclo, conforme seus idealizadores excede 2,78 $\mathrm{X} 10^{13}$.

O processo de simulação de um valor diário para a variável climática umidade relativa do ar, resume-se basicamente na geração de um número aleatório uniformemente distribuído no intervalo $[0 ; 1]$, que confrontado com a probabilidade de ocorrência de um dia chuvoso, determinará o tipo de distribuição de probabilidade a ser utilizado.

Para validação dos dados simulados em relação aos dados observados, 5 replicações do simulador foram efetuadas para a localidade avaliada, gerando séries de umidade relativa do ar com períodos distintos em relação a série histórica inicialmente parametrizada. Assim, para Ponta Grossa, foi utilizada uma série de 24 anos (1954-1977) para a parametrização dos modelos probabilísticos e outra série de 24 anos (1978-2001) para efetuar a validação. Dessa forma foram confrontados os dados simulados com dados observados que não foram utilizados no processo de parametrização, tornando os dados simulados independentes dos dados observados.

A partir de cada replicação foram computados mensalmente, os valores médios de umidade relativa da localidade avaliada. Foram realizados os testes estatísticos " $t$ ", " $F$ " e "K-S" (Kolmogorov-Smirnov), ao nível de significância de $1 \%$, para avaliar as médias e variâncias dos valores médios mensais e as formas das distribuições mensais dos dados, respectivamente. Ainda com o intuito de verificar as tendências das séries climáticas, foram realizadas análises comparativas por meio de gráficos de tendência dos valores médios mensais, do coeficiente de correlação " $r$ " de Pearson, do índice de concordância " $d$ " (WILLMOTT et al., 1981) e do índice de desempenho "c" apresentado por Camargo e Sentelhas (1997).

\section{RESULTADOS E DISCUSSÃO}

A Figura 1 apresenta a interface principal do modelo computacional para simulação de séries climáticas diárias de umidade relativa do ar, para o qual foi dado o nome "SIMIUMI" onde as quatro funções básicas são "Criar Arquivo" para uma nova localidade, "Abrir Arquivo" existente onde é possível "Importar Dados" novos ou executar efetivamente a "Simulação".

Na Figura 2 é mostrado o formato do arquivo a ser importado pelo "SIMIUMI", que deverá ser do tipo texto "separado por ponto e vírgula" (*.CSV) do Sistema Operacional Windows, onde deverão conter o campo data, o valor de precipitação pluviométrica e o valor da umidade relativa do ar. Eventualmente o valor de precipitação pluviométrica poderá ser " 0 " ou " 1 ", pois o modelo precisa apenas da informação de ocorrência de chuva. 


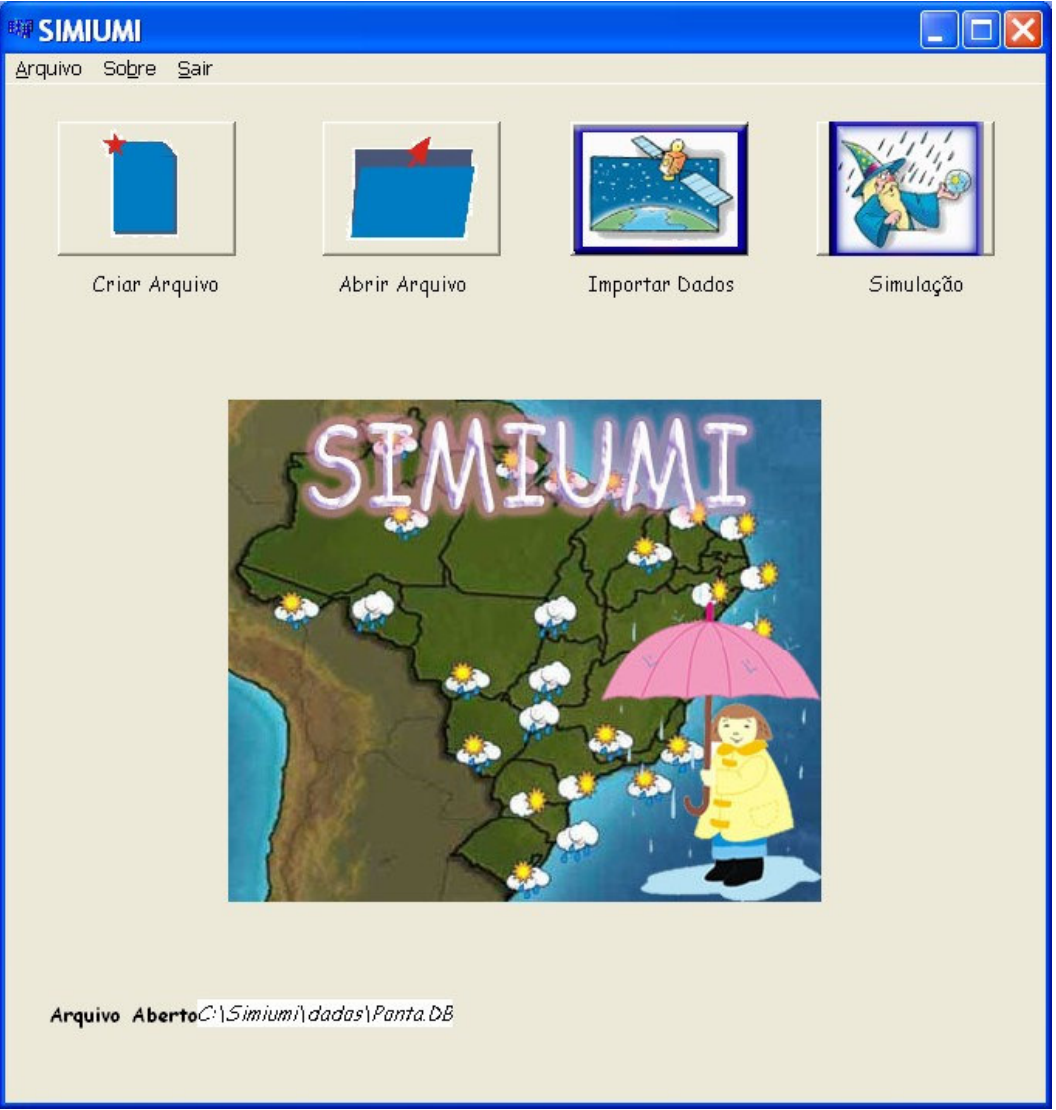

Figura 1 - Interface principal do SIMIUMI.

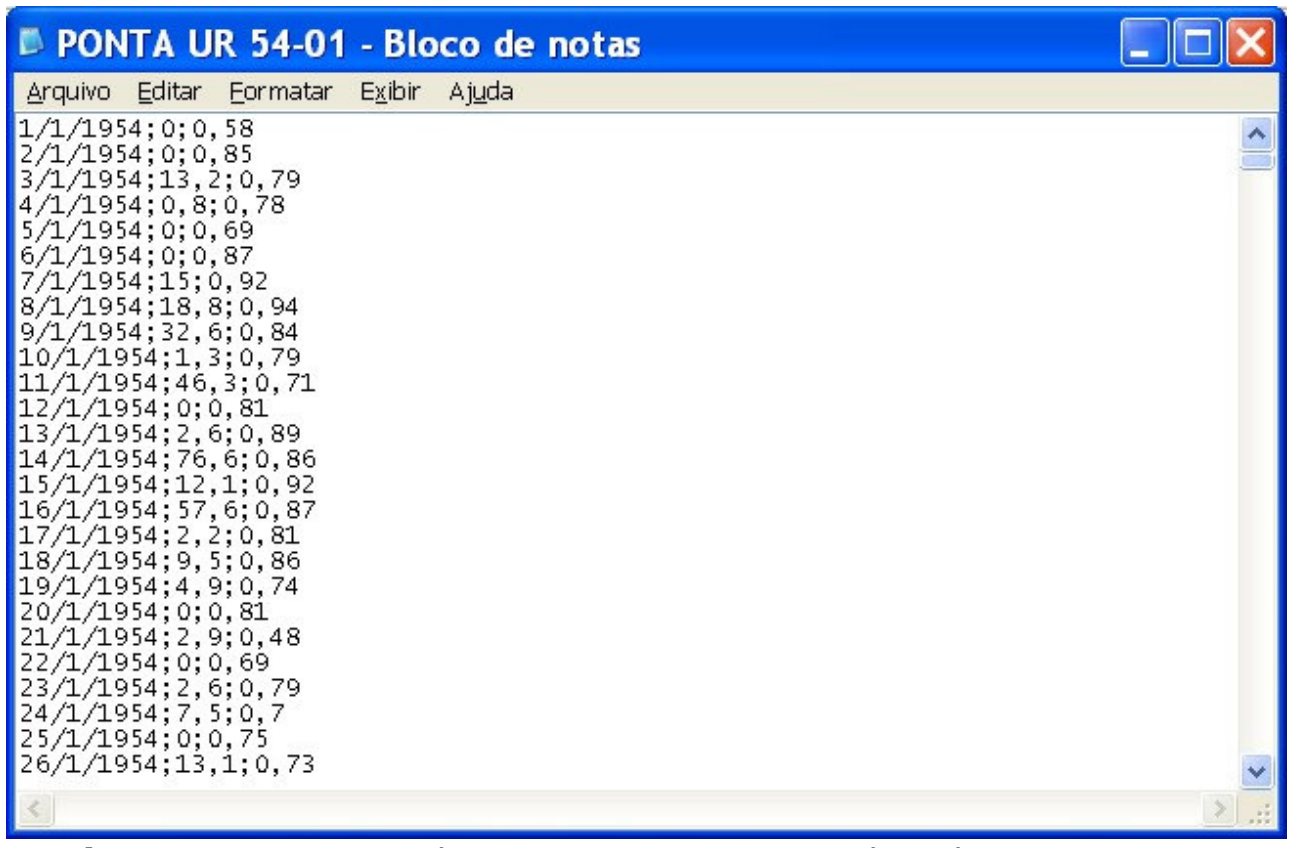

Figura 2 - Formato do arquivo a ser importado pelo SIMIUMI. 
A Figura 3 apresenta a interface de parametrização das distribuições de probabilidade, onde se percebe os valores da média e desvio-padrão (distribuição Normal) para "TUCP" e os parâmetros " $p$ " e "q" (distribuição Beta). E importante observar que a cada ano simulado estes parâmetros se alteram dinamicamente.

\begin{tabular}{|c|c|c|c|c|c|c|c|c|}
\hline \multicolumn{8}{|c|}{ 玟解 Simulação } & $-\square$ \\
\hline & MediaU & DePadU & MaxU & MinU & PS & Q5 & Markov1 & Markov2 \\
\hline Janeiro & 0.24639 & 0,07630 & 0,97000 & 0,58000 & 14,87412 & 5,31437 & 0,66270 & 0,34836 \\
\hline Fevereiro & 0,27514 & 0,06626 & 1,00000 & 0,56000 & 18,13749 & 5,68314 & 0,66667 & 0,38756 \\
\hline Mharço & 0,22012 & 0,06764 & 1,00000 & 0,62000 & 21,69808 & 6,57774 & 0,62312 & 0,30535 \\
\hline Abril & 0,24212 & 0,07333 & 0,98000 & 0,60000 & 18,15676 & 5,34773 & 0,51442 & 0,19727 \\
\hline Maio & 0,31887 & 0,08196 & 0,99000 & 0,54000 & 14,41959 & 4,31781 & 0,54458 & 0,17614 \\
\hline Junho & 0,26823 & 0,08417 & 0,99000 & 0,60000 & 11,44148 & 3,61405 & 0.51263 & 0,18487 \\
\hline Julho & 0,32345 & 0,10183 & 1,00000 & 0,51000 & 9,48360 & 3,50152 & 0,48305 & 0,16138 \\
\hline Agosto & 0,42465 & 0,10003 & 0,99000 & 0,41000 & 7,76958 & 3,25367 & 0,44321 & 0.17835 \\
\hline Setembro & 0,31578 & 0,09345 & 1,00000 & 0,54000 & 7,36774 & 3,24775 & 0,58532 & 0,25251 \\
\hline Outubro & 0,34048 & 0,09490 & 1,00000 & 0,49000 & 8,95867 & 3,67782 & 0,57000 & 0,29054 \\
\hline Novembro & 0,35889 & 0,10496 & 0,97000 & 0,44000 & 10,58734 & 4,71179 & 0,50182 & 0,30787 \\
\hline Dezembro & 0,30398 & 0,08950 & 0,96000 & 0,50000 & 11,79111 & 4,87074 & 0,55646 & 0,30936 \\
\hline \multirow{2}{*}{\multicolumn{2}{|c|}{ Periodo Base }} & o Inicial & \multicolumn{2}{|c|}{ Ano Final } & & & & \\
\hline & & $54 \div$ & 2001 & $\div$ & & & & \\
\hline \multicolumn{2}{|c|}{ Qtde Anos a Simular } & $\div$ & & & Simula & & Sair & \\
\hline \multicolumn{2}{|c|}{ Qtde Anos Simulados } & & & & & & & \\
\hline
\end{tabular}

Figura 3 - Interface de parametrização das distribuições probabilísticas.

Na Figura 4 é apresentado o formato do arquivo de saída com os dados simulados pelo "SIMIUMI", o qual é semelhante ao formato do arquivo importado pelo sistema. O detalhe é que na saída o valor da precipitação pluviométrica é " 0 " ou " 1 " indicando o estado de ocorrência da mesma, simulada pela primeira ordem da cadeia de Markov.

A Tabela 1 apresenta mensalmente o resultado do teste K-S para as 5 replicações do modelo avaliando a distribuição dos valores de umidade relativa do ar em dias secos para Ponta Grossa-PR. Verificou-se um excelente desempenho do modelo probabilístico da distribuição Beta nos meses de fevereiro, maio, julho, agosto e setembro, os quais não apresentaram significância estatística entre as distribuições simuladas e observadas. Nos meses de março, junho e outubro o modelo mostrou um desempenho satisfatório apresentando significâncias estatísticas em 2 das 5 replicações. Porém, nos meses de janeiro e abril, nos quais 4 das 5 replicações, e no mês de dezembro onde as 5 replicações apresentaram significâncias estatísticas, o modelo apresentou desempenho moderado para distribuir adequadamente os valores de umidade relativa do ar em dias secos, uma vez que para estes meses as diferenças máximas (Dmax) encontradas ultrapassaram o limite da estatística do teste K-S (D1\%) em 0,02. 


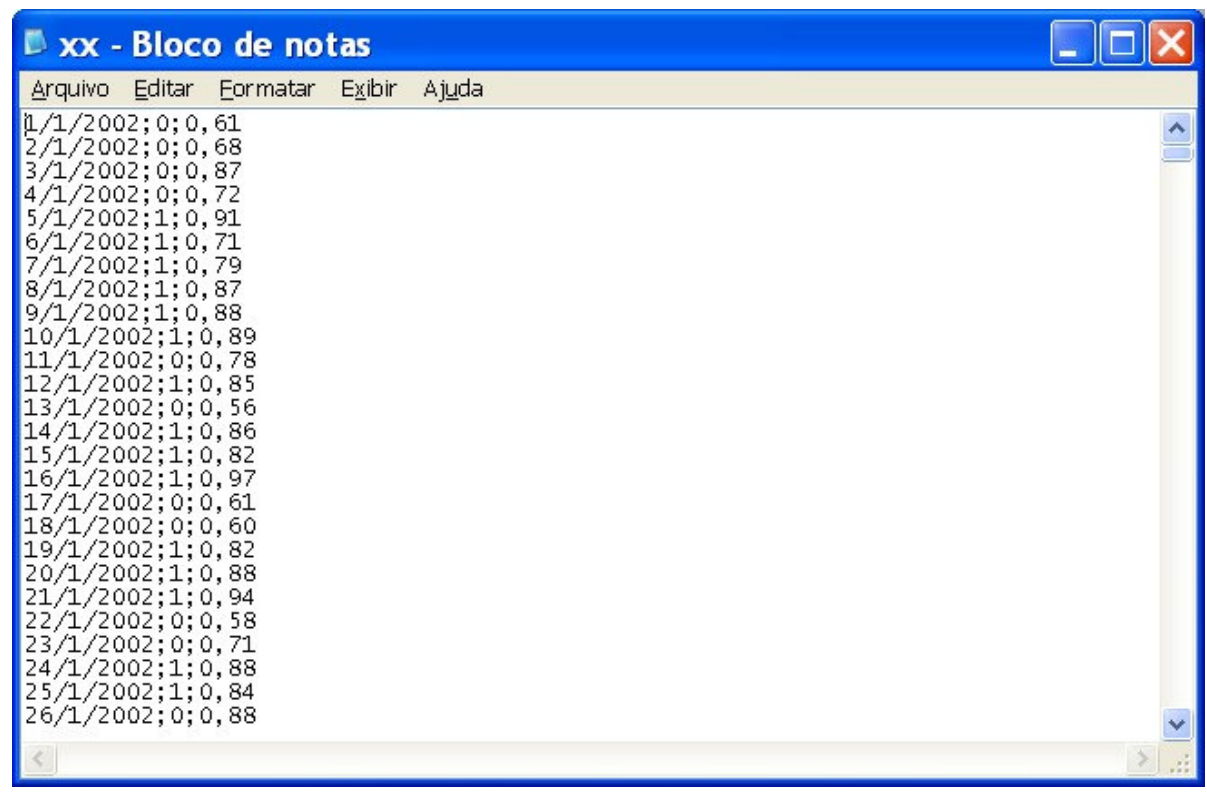

Figura 4 - Formato do arquivo de saída com os dados simulados pelo SIMIUMI.

Tabela 1 - Resultado mensal do teste K-S comparando as freqüências observadas e simuladas dos valores de umidade relativa do ar para dias secos.

\begin{tabular}{ccccccccccccc}
\hline & $\underline{\mathbf{S 1}}$ & & $\underline{\mathbf{S 2}}$ & & $\underline{\mathbf{S 3}}$ & & $\underline{\mathbf{S 4}}$ & & $\underline{\mathbf{S 5}}$ & \\
Mês & $\underline{\mathbf{D 1} \%}$ & $\underline{\text { Dmax }}$ & $\underline{\mathbf{D 1} \%}$ & $\underline{\text { Dmax }}$ & $\underline{\mathbf{D 1} \%}$ & $\underline{\text { Dmax }}$ & $\underline{\text { D1\% }}$ & $\underline{\text { Dmax }}$ & $\underline{\text { D1\% }}$ & $\underline{\text { Dmax }}$ \\
& & & & & & & & & & \\
JAN & 0,121 & $\mathbf{0 , 1 7 8}$ & 0,118 & $\mathbf{0 , 1 2 6}$ & 0,124 & $\mathbf{0 , 1 6 1}$ & 0,118 & 0,106 & 0,123 & $\mathbf{0 , 1 4 5}$ \\
FEV & 0,131 & 0,023 & 0,124 & 0,062 & 0,127 & 0,060 & 0,126 & 0,066 & 0,129 & 0,106 \\
MAR & 0,110 & 0,069 & 0,115 & 0,100 & 0,108 & $\mathbf{0 , 1 1 2}$ & 0,111 & $\mathbf{0 , 1 1 7}$ & 0,117 & 0,108 \\
ABR & 0,101 & $\mathbf{0 , 1 5 2}$ & 0,100 & $\mathbf{0 , 1 0 9}$ & 0,103 & $\mathbf{0 , 1 3 4}$ & 0,099 & 0,084 & 0,100 & $\mathbf{0 , 1 3 8}$ \\
MAI & 0,098 & 0,045 & 0,098 & 0,052 & 0,098 & 0,053 & 0,099 & 0,066 & 0,097 & 0,083 \\
JUN & 0,101 & $\mathbf{0 , 1 1 8}$ & 0,104 & 0,064 & 0,100 & 0,058 & 0,101 & $\mathbf{0 , 1 0 3}$ & 0,099 & 0,071 \\
JUL & 0,098 & 0,072 & 0,094 & 0,064 & 0,096 & 0,036 & 0,097 & 0,061 & 0,098 & 0,095 \\
AGO & 0,097 & 0,081 & 0,096 & 0,080 & 0,094 & 0,089 & 0,096 & 0,054 & 0,098 & 0,081 \\
SET & 0,104 & 0,065 & 0,107 & 0,050 & 0,105 & 0,058 & 0,106 & 0,050 & 0,105 & 0,055 \\
OUT & 0,105 & $\mathbf{0 , 1 3 6}$ & 0,106 & 0,027 & 0,108 & 0,064 & 0,107 & 0,078 & 0,108 & $\mathbf{0 , 1 3 0}$ \\
NOV & 0,110 & $\mathbf{0 , 1 3 5}$ & 0,109 & $\mathbf{0 , 1 4 0}$ & 0,111 & $\mathbf{0 , 1 6 2}$ & 0,108 & $\mathbf{0 , 1 2 5}$ & 0,108 & $\mathbf{0 , 1 2 0}$ \\
DEZ & 0,116 & $\mathbf{0 , 1 2 1}$ & 0,110 & $\mathbf{0 , 1 2 2}$ & 0,110 & $\mathbf{0 , 1 4 9}$ & 0,114 & $\mathbf{0 , 1 5 0}$ & 0,113 & $\mathbf{0 , 1 3 4}$ \\
\hline
\end{tabular}

De uma maneira mais generalizada ao longo do ano o modelo da distribuição Beta simulou satisfatoriamente as distribuições frequenciais para a umidade relativa do ar em dias secos, uma vez que $60 \%$ dos testes $\mathrm{K}-\mathrm{S}$ não apresentaram significância estatística ao nível de probabilidade empregado. É importante frisar que os dados observados utilizados para comparação com os 
simulados não fizeram parte da parametrização inicial da série para Ponta Grossa-PR.

Na Tabela 2 são mostrados mensalmente as médias e os desvios-padrões dos valores médios em dias secos, com os resultados dos testes " $t$ " e " $F$ " nas 5 replicações efetuadas. Nota-se que nos meses de janeiro a setembro, as médias simuladas e observadas não diferiram entre si mostrando que o modelo da distribuição Beta foi eficiente na reprodução dos valores médios mensais. Nos meses de outubro e novembro as médias foram reproduzidas muito satisfatoriamente, uma vez que poucas significâncias estatísticas foram encontradas pelo teste " $t$ ". Todavia, no mês dezembro, os dados simulados geraram, na maioria das replicações, médias estatisticamente diferentes das médias observadas, conforme se constata pelos valores de " $p$ ", indicando que para este mês o modelo reproduziu moderadamente os valores médios. Em geral $87 \%$ dos testes " $t$ " não apresentaram significância estatística, o que indica que o modelo da distribuição Beta, simulou adequadamente as médias mensais.

Tabela 2 - Resultado mensal do teste " $t$ " e " $F$ " confrontando as médias e variâncias observadas e simuladas de umidade relativa do ar em dias secos.

\begin{tabular}{|c|c|c|c|c|c|c|c|c|c|c|c|c|}
\hline MdOB & $\begin{array}{l}\text { JAN } \\
0.743\end{array}$ & $\begin{array}{c}\text { FEV } \\
0.775\end{array}$ & $\begin{array}{l}\text { MAR } \\
0.769\end{array}$ & $\begin{array}{l}\text { ABR } \\
0.770\end{array}$ & $\begin{array}{l}\text { MAI } \\
0.775\end{array}$ & $\begin{array}{l}\text { JUN } \\
0.763\end{array}$ & $\begin{array}{c}\text { JUL } \\
0.737\end{array}$ & $\begin{array}{l}\text { AGO } \\
0.708\end{array}$ & $\begin{array}{c}\text { SET } \\
0.701\end{array}$ & $\begin{array}{l}\text { OUT } \\
0.708\end{array}$ & $\begin{array}{l}\text { NOV } \\
0.689\end{array}$ & $\begin{array}{l}\text { DEZ } \\
0.702\end{array}$ \\
\hline DРОВ & 0.046 & 0.048 & 0.036 & 0.039 & 0.046 & 0.039 & 0.036 & 0.051 & 0.062 & 0.036 & 0.048 & 0.042 \\
\hline MdTOT & 0.755 & 0.763 & 0.776 & 0.788 & 0.783 & 0.777 & 0.749 & 0.726 & 0.709 & 0.724 & 0.716 & 0.736 \\
\hline MdS1 & 0.765 & 0.764 & 0.773 & 0.789 & 0.779 & 0.788 & 0.751 & 0.724 & 0.708 & 0.738 & 0.714 & 0.728 \\
\hline DPS1 & 0.013 & 0.020 & 0.022 & 0.017 & 0.016 & 0.024 & 0.025 & 0.022 & 0.029 & 0.027 & 0.027 & 0.029 \\
\hline$p(F) S 1$ & 0.000 & 0.000 & 0.027 & 0.000 & 0.000 & 0.026 & 0.074 & 0.000 & 0.000 & 0.171 & 0.008 & 0.088 \\
\hline$p(t) S 1$ & 0.027 & 0.308 & 0.655 & 0.035 & 0.729 & 0.011 & 0.106 & 0.174 & 0.638 & 0.002 & 0.037 & 0.017 \\
\hline MdS2 & 0.756 & 0.771 & 0.777 & 0.782 & 0.782 & 0.774 & 0.751 & 0.728 & 0.711 & 105 & 0.720 & 0.733 \\
\hline DPS2 & 0.021 & 0.017 & 0.019 & 0.016 & 0.013 & 0.029 & 0.019 & 0.024 & 0.026 & 0.028 & 0.024 & 0.025 \\
\hline$p(F) S 2$ & 0.001 & 0.000 & 0.004 & 0.000 & 0.000 & 0.155 & 0.003 & 0.001 & 0.000 & 0.248 & 0.002 & 0.013 \\
\hline$p(t) S 2$ & 0.193 & 0.744 & 0.359 & 0.168 & 0.485 & 0.275 & 0.100 & 0.101 & 0.479 & 0.712 & 0.009 & 0.004 \\
\hline MdS3 & 0.752 & 0.762 & 0.781 & 0.790 & 0.780 & 0.771 & 0.738 & 0.732 & 0.710 & 0.719 & 0.724 & 0.746 \\
\hline DPS3 & 0.025 & 0.023 & 0.017 & 0.017 & 0.013 & 0.022 & 0.027 & 0.020 & 0.024 & 0.028 & 0.023 & 0.018 \\
\hline$p(F) S 3$ & 0.005 & 0.001 & 0.001 & 0.000 & 0.000 & 0.010 & 0.210 & 0.000 & 0.000 & 0.225 & 0.001 & 0.000 \\
\hline$p(t) S 3$ & 0.366 & 0.236 & 0.146 & 0.028 & 0.669 & 0.384 & 0.936 & 0.044 & 0.540 & 0.247 & 0.003 & 0.000 \\
\hline MdS4 & 0.747 & 0.772 & 0.780 & 0.783 & 0.784 & 0.781 & 0.748 & 0.720 & 0.707 & 0.723 & 0.711 & 0.740 \\
\hline DPS4 & 0.018 & 0.023 & 0.017 & 0.017 & 0.019 & 0.021 & 0.022 & 0.024 & 0.032 & 0.024 & 0.032 & 0.025 \\
\hline p(F)S4 & 0.000 & 0.001 & 0.001 & 0.000 & 0.000 & 0.006 & 0.024 & 0.001 & 0.003 & 0.059 & 0.045 & 0.017 \\
\hline$p(t) S 4$ & 0.679 & 0.818 & 0.198 & 0.152 & 0.401 & 0.055 & 0.207 & 0.307 & 0.712 & 0.095 & 0.064 & 0.001 \\
\hline MdS5 & 0.756 & 0.745 & 0.767 & 0.794 & 0.793 & 0.770 & 0.758 & 0.724 & 0.710 & 0.736 & 0.711 & 0.735 \\
\hline DPS5 & 0.021 & 0.029 & 0.035 & 0.017 & 0.014 & 0.029 & 0.017 & 0.024 & 0.031 & 0.022 & 0.027 & 0.022 \\
\hline p(F)S5 & 0.001 & 0.020 & 0.867 & 0.000 & 0.000 & 0.183 & 0.001 & 0.001 & 0.002 & 0.019 & 0.006 & 0.003 \\
\hline$p(t) S 5$ & 0.193 & 0.013 & 0.796 & 0.011 & 0.093 & 0.473 & 0.015 & 0.195 & 0.537 & 0.003 & 0.058 & 0.002 \\
\hline
\end{tabular}


Analisando a variabilidade dos valores médios mensais em torno de suas respectivas médias mensais, representada por seus respectivos desviospadrões, percebe-se que apenas nos meses de junho, julho, outubro e dezembro o modelo da distribuição Beta gerou as variabilidades de maneira satisfatória, uma vez que poucos valores de " $p$ " do teste " $F$ " apresentaram significância estatística. Nos demais meses do ano as variabilidades simuladas não concordaram muito com as variabilidades observadas, conforme indicam os resultados do teste " $F$ ". Ao longo dos meses do ano apenas $32 \%$ dos testes " $F$ " não apresentaram significância estatística.

Em termos globais o modelo da distribuição Beta superestimou levemente as médias dos valores médios mensais em dias secos em aproximadamente $0,01(1 \%)$, enquanto que os desvios-padrões foram subestimados em torno de $0,02(2 \%)$, conforme ilustram as curvas (em vermelho) no gráfico da Figura 5. A Tabela 3 apresentando os índices " $d$ ", " $r$ " e "c" para as 5 replicações do modelo, confronta as médias observadas e simuladas entre os meses do ano, apontando que no geral o modelo foi considerado "MUITO BOM", conforme a média $(0,8330)$ do índice de desempenho " $c$ ".

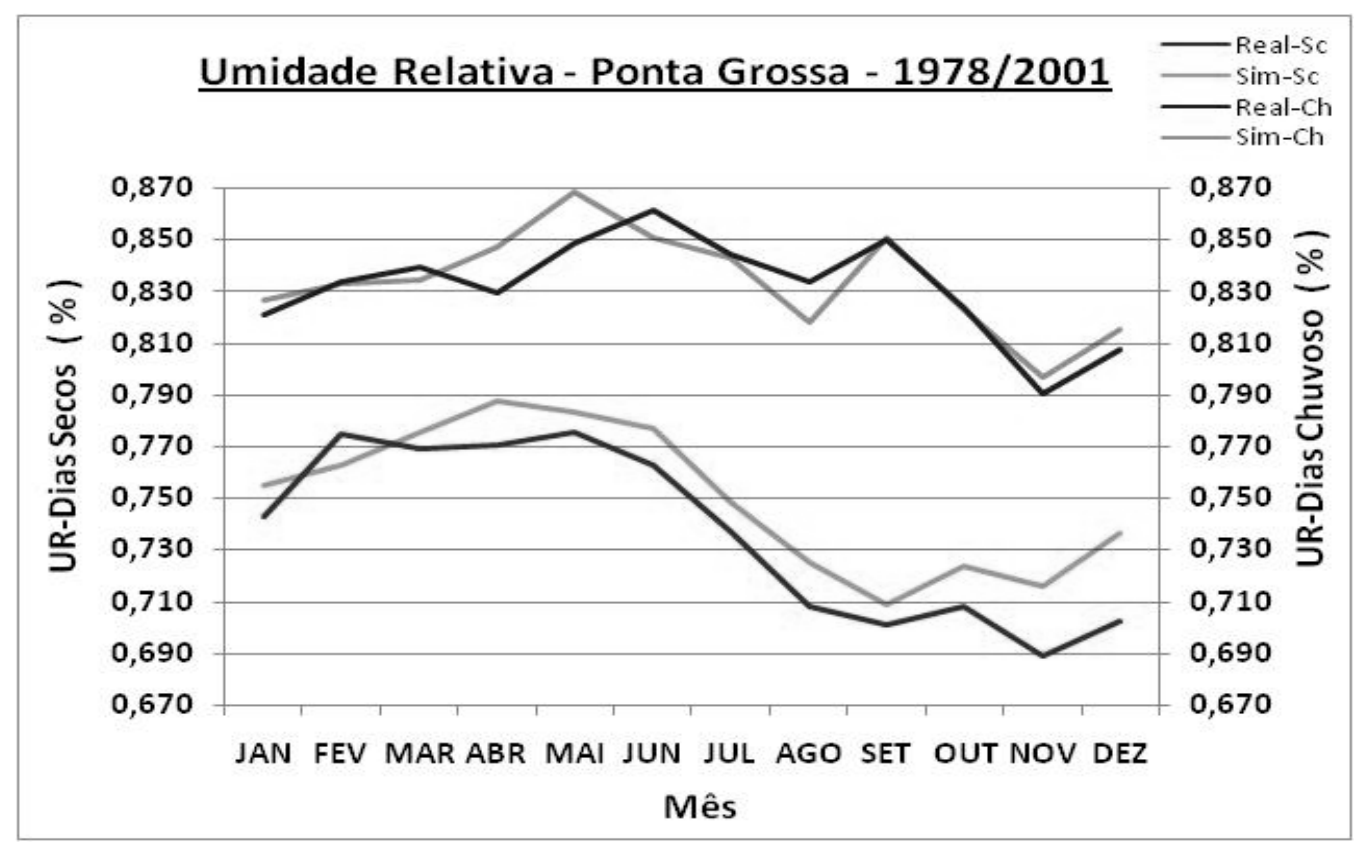

Figura 5 - Gráfico de tendência mensal da umidade relativa do ar mostrando as médias observadas e simuladas (nas 5 replicações do modelo), em dias secos e dias chuvosos.

Tabela 3 - Índices de concordância "d", de correlação "r" e de desempenho "c" para as 5 replicações do modelo, avaliando as médias mensais observadas e simuladas para dias secos entre os meses do ano.

\begin{tabular}{cccccc}
\hline & $\underline{\mathbf{S 1}}$ & $\underline{\mathbf{S 2}}$ & $\underline{\mathbf{S 3}}$ & $\underline{\mathbf{S 4}}$ & $\underline{\mathbf{S 5}}$ \\
$\mathbf{d}$ & 0.9015 & 0.9277 & 0.8836 & 0.9305 & 0.8770 \\
$\mathbf{r}$ & 0.9344 & 0.9499 & 0.8942 & 0.9548 & 0.8697 \\
$\mathbf{C}$ & 0.8423 & 0.8813 & 0.7901 & 0.8884 & 0.7627 \\
\hline
\end{tabular}


A Tabela 4 mostra mensalmente 0 resultado do teste $\mathrm{K}-\mathrm{S}$ para as 5 replicações do modelo para os valores de umidade relativa do ar em dias chuvosos, onde verificou-se um excelente desempenho do modelo da distribuição Normal para todos os meses do ano, os quais apresentaram raríssimas significâncias estatísticas entre as distribuições frequenciais simuladas e observadas, tendo em vista que os dados observados na comparação com simulados não foram usados na parametrização da simulação e, apenas $8 \%$ dos testes K-S apresentaram significância estatística ao nível de probabilidade adotado.

Tabela 4 - Resultado mensal do teste K-S comparando as freqüências observadas e simuladas dos valores de umidade relativa do ar para dias chuvosos.

\begin{tabular}{llllllllllll}
\hline & $\underline{\mathbf{S 1}}$ & & $\underline{\mathbf{S 2}}$ & & $\underline{\mathbf{S 3}}$ & & $\underline{\mathbf{S 4}}$ & & $\underline{\mathbf{S 5}}$ & \\
$\underline{\text { Mês }}$ & $\underline{\mathbf{D 1} \%}$ & $\underline{\text { Dmax }}$ & $\underline{\mathbf{D 1} \%}$ & $\underline{\text { Dmax }}$ & $\underline{\mathbf{D 1} \%}$ & $\underline{\text { Dmax }}$ & $\underline{\mathbf{D 1 \%}}$ & $\underline{\text { Dmax }}$ & $\underline{\underline{\text { D1\% }}}$ & $\underline{\text { Dmax }}$ \\
JAN & 0,118 & 0,029 & 0,122 & 0,041 & 0,115 & 0,040 & 0,123 & 0,057 & 0,116 & 0,054 \\
FEV & 0,121 & 0,087 & 0,130 & 0,045 & 0,125 & 0,050 & 0,126 & 0,082 & 0,122 & 0,029 \\
MAR & 0,132 & 0,059 & 0,125 & 0,084 & 0,139 & 0,119 & 0,131 & 0,038 & 0,123 & 0,102 \\
ABR & 0,162 & 0,034 & 0,170 & 0,065 & 0,156 & 0,095 & 0,180 & 0,052 & 0,167 & 0,096 \\
MAI & 0,174 & 0,165 & 0,169 & $\mathbf{0 , 1 8 0}$ & 0,173 & 0,113 & 0,166 & 0,153 & 0,177 & 0,123 \\
JUN & 0,162 & 0,141 & 0,154 & $\mathbf{0 , 2 1 2}$ & 0,168 & 0,142 & 0,162 & 0,161 & 0,178 & $\mathbf{0 , 2 7 4}$ \\
JUL & 0,167 & 0,109 & 0,204 & 0,139 & 0,178 & 0,140 & 0,176 & 0,110 & 0,167 & 0,095 \\
AGO & 0,175 & $\mathbf{0 , 2 2 3}$ & 0,177 & $\mathbf{0 , 2 2 7}$ & 0,208 & 0,183 & 0,176 & 0,124 & 0,170 & 0,161 \\
SET & 0,156 & 0,106 & 0,145 & 0,133 & 0,152 & 0,142 & 0,149 & 0,092 & 0,150 & 0,099 \\
OUT & 0,146 & 0,067 & 0,142 & 0,139 & 0,137 & 0,102 & 0,139 & 0,103 & 0,135 & 0,049 \\
NOV & 0,138 & 0,064 & 0,139 & 0,137 & 0,137 & 0,054 & 0,142 & 0,090 & 0,142 & 0,087 \\
DEZ & 0,124 & 0,061 & 0,133 & 0,062 & 0,132 & 0,105 & 0,126 & 0,080 & 0,128 & 0,073 \\
\hline
\end{tabular}

Na Tabela 5 são apresentados mensalmente as médias, desvios-padrões e os valores de " $p$ " dos testes " $t$ " e " $F$ " para dias chuvosos. Raramente as médias e os desvios-padrões das séries simuladas e observadas apresentaram diferenças estatisticamente significativas. Apenas no mês de fevereiro, constatou-se a não concordância dos desvios-padrões em todas as replicações, fato este que não interferiu nas médias simuladas, que segundo o teste " $t$ " foram aceitas em sua totalidade, como sendo semelhantes às médias observadas. No geral $97 \%$ dos testes " $t$ " e $82 \%$ dos testes " $F$ " não apresentaram significância estatística, mostrando que o modelo baseado na distribuição Normal foi eficiente na reprodução das médias mensais dos valores médios e suas respectivas variabilidades.

Em termo globais o modelo simulou médias e variabilidades em torno destas eficazmente, embora pequenas subestimativas da médias históricas de aproximadamente $0,01(1 \%)$ tenham ocorrido nos os meses do ano, como pode ser verificado nas curvas de tendência (em azul) apresentadas no gráfico da Figura 5. A Tabela 6 mostra os índices " $d$ ", " $r$ ", e "c" para cada uma das replicações do modelo baseado na distribuição Normal, onde se conclui pela média de 0,6715 do índice " $c$ ", que o modelo apresentou no geral um 
desempenho "BOM", quando se confrontou as médias observadas e simuladas entre os meses do ano.

Tabela 5 - Resultado mensal do teste " $t$ " e " $F$ " confrontando as médias e variâncias observadas e simuladas de umidade relativa do ar em dias chuvosos.

\begin{tabular}{|c|c|c|c|c|c|c|c|c|c|c|c|c|}
\hline 3 & $\frac{A N}{821}$ & $\frac{E V}{834}$ & $\frac{A R}{839}$ & $\frac{B R}{830}$ & $\frac{\mid A I}{849}$ & $\frac{\mathbf{J N}}{361}$ & $\frac{U L}{844}$ & $\frac{G O}{334}$ & $\frac{E T}{850}$ & $\frac{\mathbf{T}}{24}$ & $\frac{\text { OV }}{791}$ & .808 \\
\hline РОВ & 031 & & & & & & & & 1 & & 3 & .030 \\
\hline OT & 0.827 & 833 & 334 & 847 & 869 & 851 & 843 & 318 & 0.851 & 823 & 797 & 21 \\
\hline 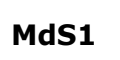 & 0.828 & 0.831 & 5 & & & 0.849 & & & & 0.840 & 9 & 818 \\
\hline DPS1 & 0.019 & 0.016 & 9 & 26 & 020 & 5 & 2 & 56 & 34 & 33 & 30 & 017 \\
\hline . & 0.021 & 0.000 & 0.359 & 0.001 & 0.001 & 0.021 & 96 & 61 & 719 & 0.987 & 996 & 008 \\
\hline - & 0.376 & 0.743 & 0.454 & 0.372 & 110 & 0.235 & 49 & 580 & 846 & 0.080 & 453 & 155 \\
\hline (10) & 0.824 & 0.833 & 0.834 & 0.843 & 0.853 & 0.854 & 0.816 & 821 & 846 & 0.812 & .778 & .816 \\
\hline & 0.024 & 0.013 & 0.020 & 0.033 & 0.025 & 0.035 & 48 & 0.059 & 24 & 0.034 & .028 & 025 \\
\hline F)S2 & 0.217 & 0.000 & 0.575 & 0.019 & 0.018 & 0.396 & 524 & 566 & 19 & 0.834 & .045 & 379 \\
\hline$(t) S 2$ & 0.778 & 0.938 & 0.388 & 0.319 & 653 & 0.501 & 33 & 418 & 40 & 0.239 & 229 & 314 \\
\hline MdS3 & 0.828 & 0.836 & 825 & 0.857 & .868 & 0.868 & 34 & 792 & 41 & 0.820 & 808 & .807 \\
\hline ב & 0.019 & 0.016 & 018 & 0.019 & 0.025 & 0.025 & 045 & 092 & 034 & 0.028 & 330 & 020 \\
\hline F)S3 & 24 & 000 & 265 & 000 & 0.014 & 0.018 & 755 & 009 & 29 & 514 & 079 & .073 \\
\hline t)S3 & 0 & 22 & 27 & 28 & .058 & 10 & 20 & 57 & 56 & 44 & 02 & 06 \\
\hline MdS4 & 335 & 0.826 & 849 & .844 & 0.881 & 0.852 & 44 & 32 & 0.862 & 0.812 & 0.789 & 0.819 \\
\hline PS4 & 0.016 & 20 & 22 & 35 & 33 & 0.028 & 0.032 & 0.035 & 0.026 & 0.031 & 0.028 & 0.017 \\
\hline 54 & 02 & 0.009 & 910 & 0.040 & 0.273 & 0.059 & 0.202 & 0.055 & 0.407 & 0.774 & 0.046 & .010 \\
\hline S4 & 0.070 & 0.363 & 134 & 0.304 & 0.005 & 0.345 & 0.992 & 64 & 59 & 0.207 & 0.839 & 125 \\
\hline MdS5 & 20 & 0 & 29 & 2 & 76 & 30 & 63 & 22 & 54 & 2 & 10 & 16 \\
\hline S5 & 8 & 0.018 & 6 & 0.036 & 2 & 0.036 & 1 & 45 & 0 & 33 & 25 & 017 \\
\hline & & 0.002 & 72 & 55 & 174 & 2 & 0.157 & 6 & 30 & 39 & 12 & .008 \\
\hline )S5 & 847 & 1 & 071 & 0. & 5 & 008 & 35 & 1 & 9 & 04 & 9 & 40 \\
\hline
\end{tabular}

Tabela 6 - Índices de concordância " $d$ ", de correlação " $r$ " e de desempenho "c" para as 5 replicações do modelo, avaliando as médias mensais observadas e simuladas para dias chuvosos entre os meses do ano.

\begin{tabular}{cccccc}
\hline & $\underline{\mathbf{S 1}}$ & $\underline{\mathbf{S 2}}$ & $\underline{\mathbf{S 3}}$ & $\underline{\mathbf{S 4}}$ & $\underline{\mathbf{S 5}}$ \\
$\mathbf{d}$ & 0.9120 & 0.9100 & 0.7941 & 0.8966 & 0.7787 \\
$\mathbf{r}$ & 0.8650 & 0.8531 & 0.6610 & 0.8528 & 0.6458 \\
$\mathbf{c}$ & 0.7888 & 0.7762 & 0.5249 & 0.7646 & 0.5029 \\
\hline
\end{tabular}

Levando-se em consideração as séries observadas e simuladas completas (dados de umidade relativa do ar em dias secos e chuvosos juntos), observouse de maneira global que modelo o computacional gerou médias muito eficientes, embora pequenas superestimativas em torno de 0,01 (1\%) tenham 
ocorrido ao longo do ano, conforme pode ser verificado na tendência apresentada no gráfico da Figura 6.

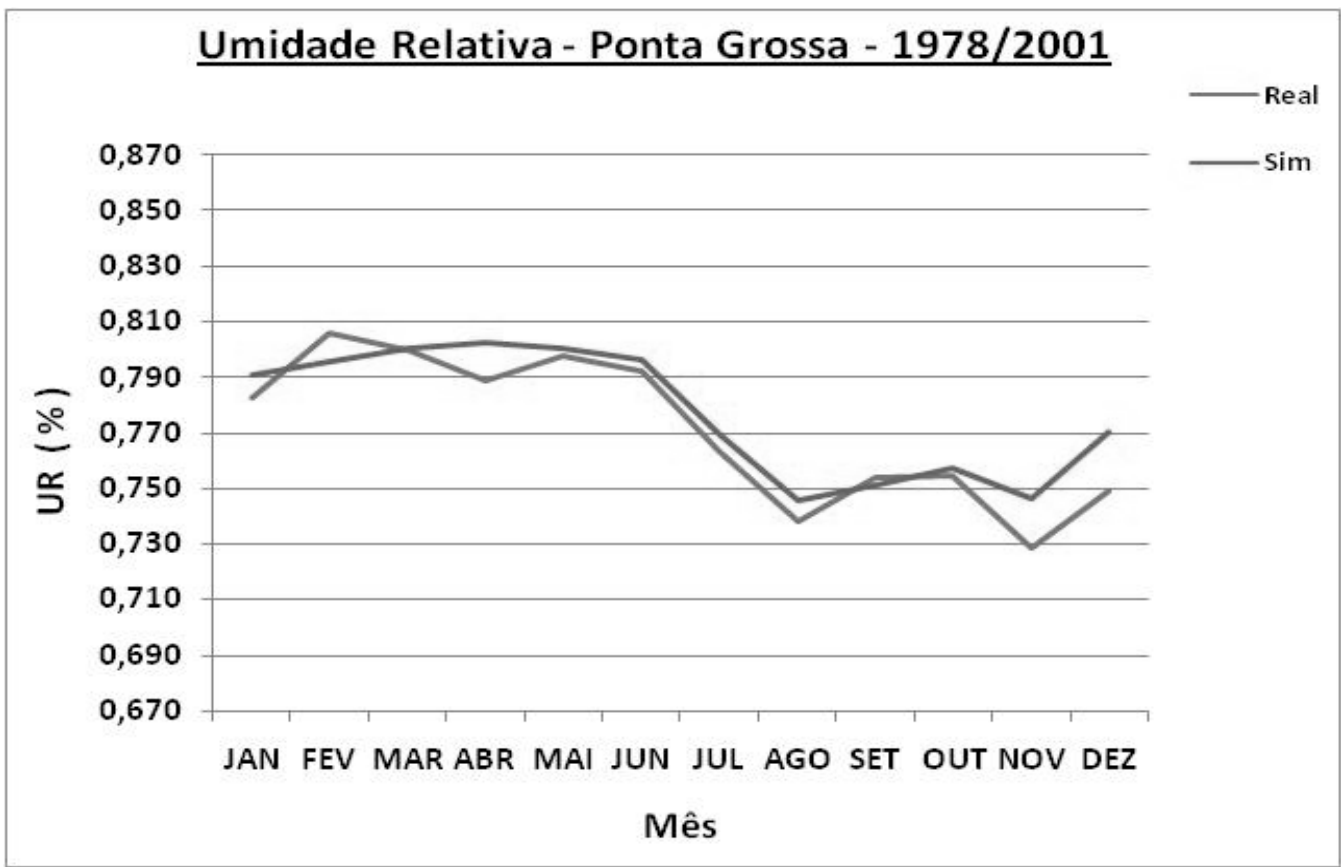

Figura 6 - Gráfico de tendência mensal da umidade relativa do ar mostrando as médias das séries climáticas completas observadas e simuladas (nas 5 replicações do modelo).

A Tabela 7 mostra os índices "d", "r", e "c", levando-se em consideração as séries observadas e simuladas completas (dados de umidade relativa do ar em dias secos e chuvosos juntos), onde se pode concluir pelo índice " $c$ " $(0,9017)$, que o modelo apresentou desempenho "ÓTIMO" na simulação de dados diários de umidade relativa do ar para Ponta Grossa-PR.

Tabela 7 - Índices de concordância "d", de correlação " $r$ " e de desempenho "c", avaliando as médias das séries climáticas completas observadas e simuladas (nas 5 replicações do modelo).

\begin{tabular}{ll}
\hline $\mathbf{d}$ & 0.9517 \\
$\mathbf{r}$ & 0.9475 \\
$\mathbf{c}$ & 0.9017 \\
\hline
\end{tabular}

É importante destacar que na simulação da umidade relativa do ar, também ficou evidenciado a tendência de subestimar a variabilidade interanual das médias, fato este que já havia sido apontado anteriormente na simulação de outras variáveis climáticas, nos trabalhos realizados por Semenov e al. (1998), Mavromatis e Hansen (2001) e Soltani e Hoogenboom (2003). O gráfico da Figura 7, mostra claramente esta tendência onde a média interanual foi gerada 
eficientemente, contudo, a variabilidade interanual em torno desta, não foi reproduzida fidedignamente.

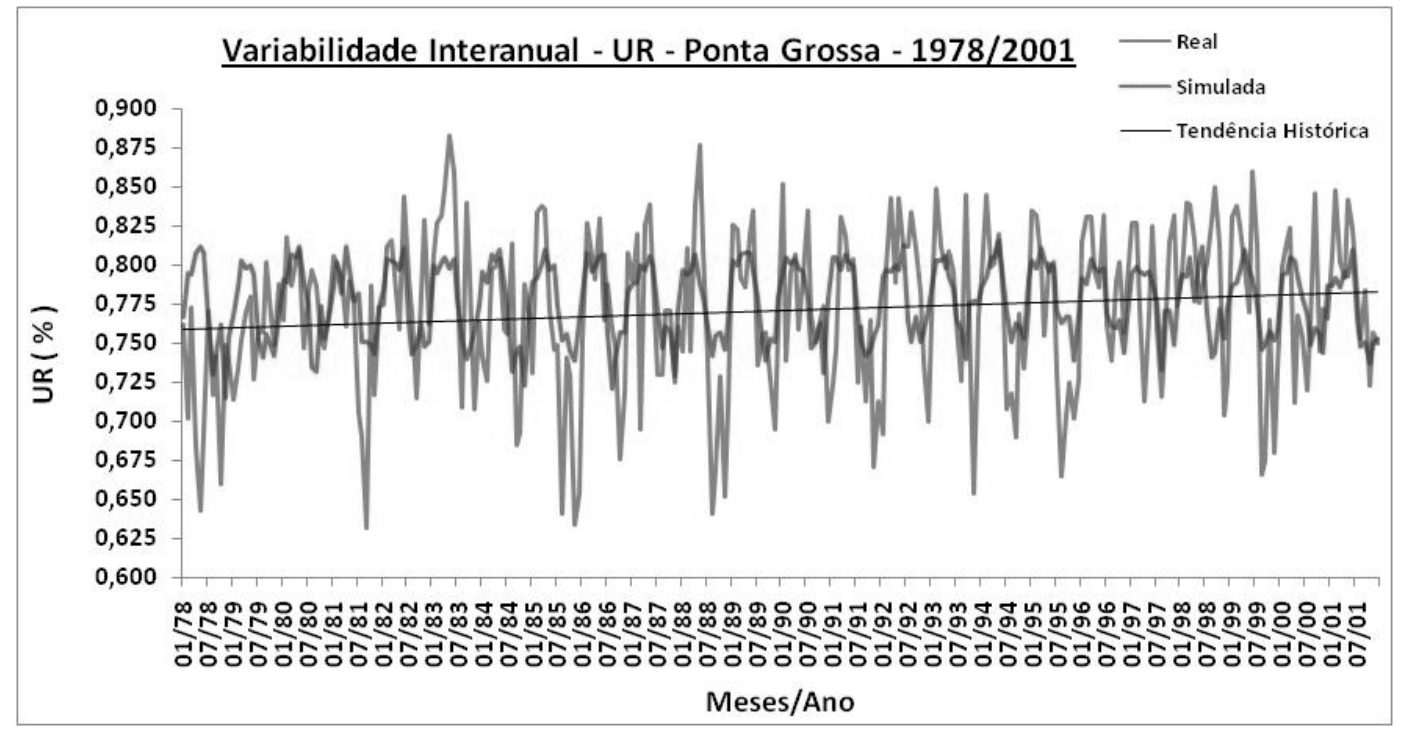

Figura 7 - Gráfico de tendência mensal da umidade relativa do ar mostrando a variabilidade interanual das médias observadas e simuladas (nas 5 replicações do modelo).

Mesmo sendo os geradores de dados climáticos uma ferramenta bastante útil na pesquisa envolvendo aspectos climáticos, é oportuno frisar, conforme alerta Hoogenboom (2000), que dados climáticos simulados só devem ser preferidos em relação aos registros históricos, quando houver a necessidade de se fazer prognósticos futuros ou quando longas séries não estiverem disponíveis e forem exigidas em modelos de tomada de decisão, ou ainda, para completar falhas de dados em séries climáticas.

\section{CONCLUSÕES}

O modelo computacional "SIMIUMI" para simulação de séries climáticas diárias de umidade relativa do ar, proposto neste trabalho, gerou dados realisticamente muito semelhantes aos dados históricos, de forma que as médias e distribuições de freqüências mensais foram bem reproduzidas pelos modelos probabilísticos da distribuição Beta (para dias considerados secos) e da distribuição Normal (para dias considerados chuvosos). Portanto, é de se esperar que quando utilizado conjuntamente em modelos bioclimatológicos, hidrológicos, de agroecossistemas ou modelos de análises de impactos ambientais, o "SIMIUMI" se apresente como uma alternativa viável em relação à registros históricos para simular a umidade do ar no cenário ambiental.

\section{AGRADECIMENTOS}

Os autores agradecem o Instituto Agronômico do Paraná - IAPAR, pela cessão dos dados climáticos utilizados neste trabalho. 


\section{REFERÊNCIAS}

BANNAYAN, M.; CROUT, N. M. J.; HOOGENBOOM, G. Application of the CERESWheat model for within-season prediction of winter wheat yield in the United Kingdom. Agronomy Journal, Madison, v.95, p.114-25, 2003.

BELTRÃO, N. E. M.; ARAÚJO, A. E.; BENASSI, A.C.; AMARAL, J. A. B.; SEVERINO L. S.; CARDOSO, G. D. Zoneamento e época de plantio para o algodoeiro no norte do estado do Espírito Santo. Revista Brasileira de Engenharia Agrícola e Ambiental, Campina Grande, v.7, n.1, p. 99-105, 2003.

BUSTOS, O.H.; FRERY, A.C. Simulação Estocástica : Teoria e Algoritmos. Rio de Janeiro: IMPA-Instituto de Matemática Pura e Aplicada, 1992. 148p.

CALIMAN, F. R. B.; SILVA, D. J. H.; FONTES, P. C. R.; STRINGHETA, P. C.; MOREIRA, G. R.; CARDOSO, A. A. Avaliação de genótipos de tomateiro cultivados em ambiente protegido e em campo nas condições edafoclimáticas de Viçosa. Horticultura Brasileira, Brasília, v.23, n.2, p.255-9, 2005.

CAMARGO, A. P.; SENTELHAS, P. C. Avaliação do desempenho de diferentes métodos de estimativa da evapotranspiração potencial no estado de São Paulo, Brasil. Revista Brasileira de Agrometeorologia, Santa Maria, v.5, n.1, p.8997, 1997.

COLA G.; FAILLA O.; MARIANI L. BerryTone-A simulation model for the daily course of grape berry temperature. Agricultural and Forest Meteorology, Amsterdam, n.149, p. 1215-28, 2009.

COSTA NETO, P. L. Estatística. São Paulo: Edgard Blucher, 1977. 264p.

HOOGEMBOOM, G. Contribution of agrometeorology to the simulation of crop production and its applications. Agricultural and Forest Meteorology, Amsterdam, n.103, p. 137-57, 2000.

KUCHAR, L. Using WGENK to generate synthetic daily weather data for modelling of agricultural processes. Mathematics and Computers in Simulation, Amsterdam, v.65, p.69-75, 2004.

LARSEN, G. A.; PENSE, R. B. Stochastic simulation of daily climatic data for agronomic models. Agronomy Journal, Madison, v.74, p. 510-4, 1982.

MAVROMATIS, T.; HANSEN, J. W. Interannual variability characteristics and simulated crop response of four stochastic weather generators. Agricultural and Forest Meteorology, Amsterdam, v.109, p.283-296, 2001.

MOTA, F. S. Meteorologia Agrícola. 5. ed., São Paulo: Nobel, 1981. 376 p.

NEIVA, J. N. M.; TEIXEIRA, M.; TURCO, S. H. N.; OLIVEIRA; S. M. P.; MOURA, A. A. A. N. Efeito do Estresse Climático sobre os Parâmetros Produtivos e Fisiológicos de Ovinos Santa Inês Mantidos em Confinamento na Região 
Litorânea do Nordeste do Brasil. Revista Brasileira de Zootecnia, v.33, n.3, p.668-78, 2004.

RICHARDSON, C. W. Weather simulation for crop management models. Transactions of the ASAE, St. Joseph, v.28, n.5, p.1602-6, 1985.

RICHARDSON, C. W.; WRIGHT, D. A. WGEN: A model for generating daily weather variables. USDA-ARS ARS-8, Washington-DC: USDA, 1984, 80p.

SEDIYAMA, G. C.; CHANCELLOR, W. J.; BURKHARDT, T. H.; GOSS, J. R. Simulação de parâmetros climáticos para a época de crescimento das plantas. Revista Ceres, Viçosa, v.25, n.141, p.455-66, 1978.

SEMENOV, M. A.; BARROW, E. M. Use of a sthochastic weather generator in the development of climate change scenarios. Climatic Changes, Norwich, v.35, p.397-415, 1997.

SEMENOV, M. A.; BROOKS, R. J.; BARROW, E. M.; RICHARDSON, C. W. Comparison of the WGEN and LARS-WG stochastic weather generators for diverse climates. Climate Research, Norwich, v.10, p. 95-107, 1998.

SHANNON, R. E. Systems simulation : the art and science. New Jersey: Prentice-Hall, 1975. 387p.

SOLTANI, A.; HOOGENBOOM, G. A statistical comparison of the stochastic weather generators WGEN and SIMMETEO. Climate Research, Norwich, v.24, p.215-30, 2003.

SOUZA, C. G.; SANT'ANNA NETO, J. L. Ritmo climático e doenças respiratórias: interações e paradoxos. Revista Brasileira de Climatologia, Rio de Janeiro, n.3, p. 65-82, 2008.

TOLENTINO M.; ROCHA-FILHO, R. C.; SILVA, R. R. A atmosfera terrestre. São Paulo: Editora Moderna, 2004. 160 p.

TURCO, S. H. N.; SILVA, T. G. F.; SANTOS, L. F. C.; RIBEIRO, P. H. B; ARAÚJO, G. G. L; JUNIOR, E. V. H; AGUIAR, M. A. Zoneamento bioclimático para vacas leiteiras no estado da Bahia. Revista de Engenharia Agrícola, Jaboticabal, v.26, n.1, p. 20-27, 2006.

VIRGENS FILHO, J. S. Modelo computacional para simulação de dados climáticos. 1997. 86 p. Dissertação (Mestrado em Agronomia/Energia na Agricultura) - Faculdade de Ciências Agronômicas, Universidade Estadual Paulista, 1997.

VIRGENS FILHO, J. S. Ferramenta computacional para simulação de séries climáticas diárias, baseada na parametrização dinâmica das distribuições de probabilidade. 2001. 92 p. Tese (Doutorado em Agronomia/Energia na Agricultura) - Faculdade de Ciências Agronômicas, Universidade Estadual Paulista, 2001. 
WICHMANN, B.A.; HILL, I.D. An efficient and portable pseudo-random number generator. Applied Statistics, v.31, p.188-90, 1982.

WILLMOTT, C. J. On the validation of models. Physical Geography, Silver Spring, v.2, n.2, p.184-94, 1981.

YAO, A.Y.M. A statistical model for the relative humidity. Journal of Applied Meteorology, v.13, p.17-21, 1974. 